\title{
YOUNG DRIVER DISTRACTION BY TEXT MESSAGING: A COMPARISON OF THE EFFECTS OF READING AND TYPING TEXT MESSAGES IN CHINESE VERSUS ENGLISH
}

\author{
Wei YAN ${ }^{\mathrm{a} *}$, S.C. WONG ${ }^{\mathrm{a}}$, Y.C. LI ${ }^{\mathrm{a}}$, N.N. SZE ${ }^{\mathrm{b}}$, Xuedong YAN \\ ${ }^{a}$ Department of Civil Engineering, The University of Hong Kong, Pokfulam Road, Hong \\ Kong, China \\ ${ }^{b}$ Department of Civil and Natural Resources Engineering, University of Canterbury, \\ Christchurch, New Zealand \\ ${ }^{c}$ Key Laboratory of Ministry of Education - Urban Transportation Complex Systems Theory \\ and Technology, School of Traffic and Transportation, Beijing Jiaotong University, China \\ * Corresponding author: Tel.: +852 2859-2662, Fax: +852 2517-0124, E-mail: \\ maggievi@hku.hk
}

\begin{abstract}
Background: Reading and typing text messages while driving causes remarkable impairments in driving performance and are prohibited in many jurisdictions. Hong Kong is a bilingual society and many people write in both Chinese and English. As the input methods of Chinese and English vary considerably, this study aimed to examine the effects of reading and typing Chinese and English text messages on driving performance via a driving simulator approach. Method: Experiments were conducted to analyze the differences in reaction time (RT), driving lane undulation (DLU), driving speed fluctuation (DSF) and car-following distance (CFD) between test and leading cars. The driving performance of 26 participants was monitored according to the following conditions: (1) no distraction, (2) reading and typing Chinese text messages and (3) reading and typing English text messages. Results: RT, DLU and DSF were significantly impaired by reading and typing both Chinese and English text messages. Moreover, typing text messages distracted drivers more than reading them. Although the Chinese text messaging input system is more complicated, the languages did not differ significantly in their degree of distraction. Conclusion: Both reading and typing text messages while driving should be prohibited regardless of whether Chinese or English is used.
\end{abstract}

Keywords: Traffic Safety, Driving Distraction, Driving Simulator, Text Messaging

\section{INTRODUCTION}

Distractions are among the main causes of road traffic accidents, and can increase the chance that an accident will occur by two to nine times compared with no distraction (Redelmeier and Tibshirani, 1997; Violanti and Marshall, 1996). Research has demonstrated that mobile phone usage while driving increases drivers' mental workload (Patten et al., 2004; Makishita and Matsunaga, 2008; Drews et al., 2009), distracts their attention, increases their reaction time delay (Al-Darrab et al., 2009; Hosking et al., 2009; Consiglio et al., 2003), impairs driving maintenance by increasing the deviation in the vehicle's lateral position and increases traffic violations by influencing the driver to speed and run stop signs (Törnros and Bolling, 2005; Hosking et al., 2009; Drews et al., 2009; Beede and Kass, 2006). It therefore increases the risk of traffic conflicts and crashes.

Numerous studies have concluded that text messaging while driving is risky (Hallett et al., 2012; Young et al., 2014; Nemme and White, 2010; Harrison, 2011; Owens et al., 2011). Text 
messaging impairs a driver's cognition, decision-making ability and ability to maneuver safely, the driver's reaction time (RT), driving lane undulation (DLU) and driving speed fluctuation (DSF) are effective indicators of impaired performance due to distraction, as shown in Table 1.

\section{[Insert Table 1 here]}

Text messaging is the primary form of communication among college students (Chiang et al., 2002), as it supports peer-to-peer interaction and increases feelings of belonging, and $98 \%$ of young drivers have texted while driving, regardless of the circumstances (Atchley et al., 2011). Many young people text rather than talk while driving (Goodwin et al., 2012). Another study of U.S. college students revealed that $91 \%$ of frequent drivers have texted while driving, and that a considerable proportion were travelling with passengers at the time (Harrison, 2011). One Australian study conducted a follow-up survey of university students after a short training session and found that texting was still prevalent, despite the students being aware that it was dangerous and illegal (Nemme and White, 2010). Another attitudinal survey of young drivers revealed that a decrease in mindfulness increased the prevalence of texting while driving, and that this association was mediated by emotion-regulation motives (Feldman et al., 2011). Additionally, in a nationwide online survey in New Zealand conducted by Hallett (2012), younger drivers are more likely to engage in reading and texting messaging, as age was found to be an important indicator of participant's willingness. Therefore, research related to the factors that increase the prevalence of young people who text message while driving is essential to developing effective remedial measures that combat this behavior.

Legislation and enforcement measures have been introduced in response to this high risk to decrease the number of road crashes, involving a deterrence theory approach to minimize unsafe driving behavior of text message while driving. The use of hand-held phones while driving has been prohibited in Hong Kong since July 1, 2000 (Road Safety Council, 2003). In one mobile phone distraction study, a driver's attitude was found to be the most consistent predictor of his or her intention to use a mobile phone while driving (Walsh et al., 2008). A before-and-after study of the introduction of hand-held mobile phone legislation in New York State revealed that 46 out of 62 counties experienced a reduction in fatal road crashes, and that all of the counties experienced a remarkable reduction in road casualties after the legislation was implemented (Nikolaev et al., 2010). However, the deterrence-based traffic law enforcement approach did not always work, and drivers continue to read and type text messages while driving. Ray (2004) suggests the legislation of mobile phone usage while driving prohibition in a more certain, swift, and severe way in order to be effective the results based on deterrence theory. A questionnaire survey in China studies the personality factors' correlations on driving behaviors, the results presented that deterrence did not affect distracted driving (Nan et al., 2011). Harrison (1998) has also argued that deterrence-based approaches may not always be informed by psychological theory and that the impact of enforcement on driving behavior. And in some cases the usage of the mobile phone even tends to increase in the short term (McCartt et al., 2006). Additionally, a study of Kansas drivers (Nelson et al., 2009) found that driver's tendencies to talk on the phone and text message while driving remained high even after the implementation of relevant legislation. Moreover, some convicted drivers reported simultaneously engaging in other risky driving behavior such as speeding, running stop signs and changing lanes carelessly (Beck et al., 2007; Harrison, 2011; Owens et al., 2011). 
Hong Kong is a bilingual metropolis, and Chinese and English are common languages used in reading and typing messages. However, because Chinese (shape based) and English (Latin alphabet based) characters are remarkably different in terms of their formation, text messaging in the two languages may make different cognitive demands of drivers and cause them to maneuver differently. Psychology and linguistic studies have observed that reading different languages requires different mental workloads. The speeds at which one reads English and Chinese can differ and require different reaction processing times. When a user inputs a Chinese text message, he or she is required to select the correct characters from a list of homophones, as several different characters can have the same pronunciation. Inputting Chinese is more complicated than inputting English, as it requires the additional step of character selection. Therefore, analyzing the differential effects of text messaging in Chinese and English on driving performance is worthwhile. The results may shed light on the extent of this problem in both Chinese and English societies.

Driving simulator experiments have increasingly been used to safely and ethically model the relationship between traffic safety and driver behavior and especially prohibited behavior in hazardous situations. Studies have covered various topics including red light jumping, incident perception and young and novice driver behavior (Yan et al., 2009; Yan et al., 2007; Auberlet et al., 2012; Bella, 2008; van Driel et al., 2007; Yang et al., 2013; Feldman et al., 2011). The driving simulator approach has been prevalent in distracted driving studies (McCartt et al., 2006), especially in studies of mobile phone usage while driving, which have considered conversation (Consiglio et al., 2003; Törnros and Bolling, 2005; Beede and Kass, 2006), dialing and text messaging (Horrey and Wickens, 2006; Hosking et al., 2009; Drews et al., 2009; Rudin-Brown et al., 2013; Young et al., 2014). In the current study, driving simulation was conducted to model the effects of reading and typing text messages on the driving performance of young drivers. Data reflecting driving performance under various conditions were collected, and the different performance measures including RT, DLU, DSF and CFD were compared. The demographic effects were also studied. Based on data analysis, the distraction measures of Chinese and English reading and typing messages are discussed in this paper.

\section{DATA}

\subsection{Participants}

Twenty-six Chinese young drivers (nineteen males and seven females) aged between 22 and 33 years $($ mean $=24.2$, S.D. $=2.5$ ) were recruited from the university for this simulation study. The driver gender ratio is based on the census result of working population with fixed place of work in Hong Kong by the transport mode private car / passenger van and the Hong Kong young driver (age 18-30) accident number proportion by the report of Women and Men in Hong Kong - Key Statistics (2014). All of the participants possessed valid full driving licenses, ranging between 6 months and 11 years $($ mean $=3.9$, S.D. $=2.3$ ). Most of the participants were occasional drivers who drove for 2.2 hours per week on average (S.D. = 3.1). The participants' demographic information is summarized in Table 2. Among the participants, $20 \%$ of the female participants and $55 \%$ of the male participants had engaged in text messaging while driving. All of participants were proficient in reading and writing both simplified Chinese and English.

[Insert Table 2 here] 
The Human Research Ethics Committee for Non-Clinical Faculties of The University of Hong Kong approved the simulated driving experiment. The purpose and experimental procedures were explained to the participants, and informed consent was obtained before the simulation study.

\subsection{Subjects/Apparatus}

\subsubsection{Driving Simulator and Driving Tasks}

A desktop-based driving simulator (XPDS 300 Driving Simulator, Version 1.6) comprising a driving scenario engine, three 19" LCD monitors and a Logitech G27 steering wheel and foot pedal control kit was used in the experiment. Driving performance data related to the vehicle's position, travel speed, acceleration and braking performance and the angle of steering wheel were recorded in a $30-\mathrm{Hz}$ sampling frame.

To monitor the distraction imposed by reading or typing text messages on an urban road, the testing scenario tested the driver's ability to maintain a safe distance from a leading vehicle at a prescribed speed of $50 \mathrm{~km} / \mathrm{h}$ along a straight road section. In the test, the leading vehicle accelerates to the prescribed speed, and then maintains this prescribed speed on the road. The driver of the test car is required to follow this leading car at a safe distance. When the leading vehicle began to brake (indicated by its rear brake light), the participant had to perform an emergency brake to a complete stop. The braking time of the leading vehicle was randomly generated to avoid interference from the learning effect. Figure 1 illustrates the testing scenario of the driving simulator in this study.

\section{[Insert Figure 1 here]}

\subsubsection{Mobile Phone and Typing Method}

The drivers were provided with a typical touchscreen-based Android smartphone, the Samsung Galaxy S, to read and type the text messages. The smartphone had a keyboardbased alphabetic English input system and a Pinyin phonetic simplified Chinese input system, which are currently the most common input methods (Chiang et al., 2002).

The text messages were chosen from a textbook that was written in English and translated into Chinese. The message content was matched exactly between the two languages. The message vocabularies of both the Chinese and English versions were in common usage. After reading the messages during the practice and experiment test sessions, all of the participants affirmed that both sets of content were understandable and that the translation was exact.

\subsection{Experimental Procedures}

The driving simulator experiment consisted of four phases, as illustrated in Figure 2.

[Insert Figure 2 here]

In Phase 1, an invigilator explained the purpose and experimental procedures to the participants, who were asked to sign the informed consent form before the experiment. Moreover, the participants' demographic information including their ages, genders, education levels, driving habits and driving experience were obtained via a driver behavior 
questionnaire.

In Phase 2, each participant took part in a 15-minute practice session to familiarize themselves with the English and Chinese input systems using the adopted smartphone. Each individual's ability to read and type text messages in both Chinese and English was then assessed.

In Phase 3, each participant took part in a 20-minute training session to familiarize themselves with the operation of the driving simulator and mobile phone. The participants were asked to drive in the simulator with no distractions. They were then asked to text message using both the English and Chinese input methods while driving.

In Phase 4, the participants were asked to perform a series of simulated driving tests under different distraction conditions: (i) no distraction (ND), (ii) reading Chinese text messages (RC), (iii) reading English text messages (RE), (iv) typing Chinese text messages (TC) and (v) typing English text messages (TE). The contents of the text messages were made the same in both languages for all of the participants to induce the same level of distraction. To further minimize the random effect, each of the driving tests was repeated twice.

In each of the tests, information related to the subject's reaction and typing times (except under the no-distraction condition) were recorded. Under the distraction conditions, messages were sent to the smartphone throughout the driving task to ensure continuous and uninterrupted distribution. The participants were asked to read and type text messages continuously during the entire driving experiment, i.e., from the time they started the test car until the appearance of the leading car's brake light. The messages were written in either English or simplified Chinese.

\subsection{Data Collection and Measures}

The participants' driving performance was measured according to RT, DLU, DSF and CFD under different distraction conditions, including ND, RC, RE, TC and TE.

(a) Reaction time (RT)

RT is measured as the length between the appearance of a leading car's brake light and the time at which a test car driver applies the brake. Hence, it includes the participant's perception and action times. In this study, as each experiment was repeated twice, RT was taken as the average value obtained from the two repeated experiments.

(b) Driving lane undulation (DLU)

DLU, DSF and CFD involved sampling the period from the time at which the test car caught up with the prescribed speed of the leading car at $50 \mathrm{~km} / \mathrm{h}$ until the time at which the participant applied the brake. The sampling interval was taken as 1/30 sec.

DLU measures the variation in a test car's lateral movement while driving, and represents the ability of the driver to stay in the lane. The road in this testing scenario is set as a perfectly straight course, and the leading car is set in the central line of this road during the entire experiment. 
It is evaluated as the standard deviation of the lateral movement away from the habitual lane position at different sampling times along the driveway, and can be expressed as follows:

$$
\sigma_{X}=\sqrt{\frac{\sum_{i=1}^{N}\left(x_{i}-\bar{x}\right)^{2}}{N}},
$$

where $\sigma_{x}$ is the DLU, $x_{i}$ is the lateral position at sampling time $i, \bar{x}=\frac{1}{N} \sum_{i=1}^{N} x_{i}$ is the mean lateral position during the sampling period and $N$ is the number of sampling points. In this study, as each experiment was repeated twice, DLU was taken as the average value obtained from the two repeated experiments.

(c) Driving speed fluctuation (DSF)

DSF measures the fluctuation of a test car's speed while driving along a lane, and represents its ability to follow a leading car steadily. It is evaluated as the standard deviation of the speed away from the average speed at different sampling times along the driveway, and can be expressed as follows:

$$
\sigma_{v}=\sqrt{\frac{\sum_{i=1}^{N}\left(v_{i}-\bar{v}\right)^{2}}{N}},
$$

where $\sigma_{v}$ is the DSF, $v_{i}$ is the speed of the test car at sampling time $i$ and $\bar{v}=\frac{1}{N} \sum_{i=1}^{N} v_{i}$ is the average speed of the test car during the sampling period. In this study, as each experiment was repeated twice, DSF was taken as the average value obtained from the two repeated experiments.

(d) Car-following distance (CFD)

CFD is measured as the space between a leading car and a test car while moving along a driveway, and represents the test car driver's ability to follow the leading car, driving preference and response to the leading car's performance. It is evaluated as the average distance between the two vehicles at different sampling times along the driveway, and can be expressed as follows:

$$
\bar{d}=\frac{\sum_{i=1}^{N} d_{i}}{N},
$$

where $\bar{d}$ is the average CFD and $d_{i}$ is the distance between the test and leading cars at sampling time $i$. In this study, as each experiment was repeated twice, CFD was taken as the average value obtained from the two repeated experiments.

Table 2 shows a summary of the collected data. The average RT is 1.09 sec with a standard deviation of $0.52 \mathrm{sec}$. DLU is 0.20 (S.D. $=0.22$ ) $\mathrm{m}$. As the expected speed is $50 \mathrm{~km} / \mathrm{h}$, the average DSF is 3.88 (S.D. $=2.38) \mathrm{km} / \mathrm{h}$, representing a less than $10 \%$ fluctuation in variance. The average CFD of the collected data is 37.49 (S.D. = 8.49) $\mathrm{m}$. 


\section{METHOD}

\subsection{Driving Performance Factor}

To neutralize the demographic effect of the participants, a driving performance factor $f_{k i}$ was defined as the ratio between the participants' driving performance with and without distraction. The driving performance factor is formulated as follows:

$$
f_{k i}=\frac{y_{k i}}{y_{0 i}}
$$

where $y$ measures the RT, DLU, DSF or CFD of a participant $i . k$ is the distraction type, with $k=1,2,3$ and 4 representing the RC, RE, TC and TE distractions, respectively. The performance factor could be interpreted as the driving performance of participant $i$ with a distraction type $k$ that increases the performance measure by $\left(f_{k i}-1\right) \times 100 \%$ compared with the no-distraction condition.

The mean $\mu_{k}$ and standard deviation $\sigma_{k}$ of the performance factor for distraction type $k$ were obtained for the participants as follows:

$$
\begin{gathered}
\mu_{k}=\frac{\sum_{i=1}^{M} f_{k i}}{M} \text { and } \\
\sigma_{k}=\frac{1}{\sqrt{M}} \sqrt{\frac{\sum_{i=1}^{M}\left(f_{k i}-\mu_{k}\right)^{2}}{M-1}},
\end{gathered}
$$

where $M$ is the number of participants.

Assume that the performance factor for distraction type $k$ follows a lognormal distribution with a mean $\mu_{k}$ and a standard deviation $\sigma_{k}$. The parameters of this lognormal distribution can be determined by the expressions in the Appendix. Denote $\mathrm{H}_{0}$ as the null hypothesis that distraction $k$ has no effect on the performance measure $f_{k}$. From this lognormal distribution, the $95 \%$ confidence interval $\left(f_{k}^{l}, f_{k}^{u}\right)$ can be identified, with $\operatorname{Pr}\left\{f_{k} \leq f_{k}^{l}\right\}=\operatorname{Pr}\left\{f_{k} \geq f_{k}^{u}\right\}=2.5 \%$. If $f_{k}^{l} \leq 1 \leq f_{k}^{u}$, there is insufficient evidence to reject the null hypothesis. Otherwise, $\mathrm{H}_{0}$ can be rejected, and it can be concluded that distraction $k$ affects the performance measure by $\left(\mu_{k}-1\right) \times 100 \%$ at the $5 \%$ significance level.

\subsection{Cross-Comparison Distraction Factor of Driving Performance}


Different driving performance factors were directly compared to further ascertain the relative effects of different distractions. The cross-comparison distraction factor of two different distraction effects is specified as follows:

$$
\xi_{j k i}=\frac{f_{j i}}{f_{k i}}=\frac{y_{j i} / y_{0 i}}{y_{k i} / y_{0 i}}=\frac{y_{j i}}{y_{k i}},
$$

which represents the relative effect of distraction type $j$ compared with distraction type $k$ for individual $i$, indicating that the distraction effect of type $j$ on the performance measure is $\left(\xi_{j k i}-1\right) \times 100 \%$ greater than the distraction effect of type $k$ on individual $i$.

The mean cross-comparison ratio $\pi_{j k}$ and the standard deviation $\omega_{j k}$ for distraction types $j$ and $k$ can be estimated for the participants as follows:

$$
\begin{gathered}
\pi_{j k}=\frac{\sum_{i=1}^{M} \xi_{j k i} \text { and }}{M} \\
\omega_{j k}=\frac{1}{\sqrt{M}} \sqrt{\frac{\sum_{i=1}^{M}\left(\xi_{j k i}-\pi_{j k}\right)^{2}}{M-1}} .
\end{gathered}
$$

Assume that the cross-comparison ratio follows a lognormal distribution with a mean and a standard deviation of $\pi_{j k}$ and $\omega_{j k}$, respectively. Denote $\mathrm{H}_{1}$ as the null hypothesis that there is no difference between the relative effects of distraction types $j$ and $k$ on the performance measure. The $95 \%$ confidence interval $\left(\xi_{j k}^{l}, \xi_{j k}^{u}\right)$ can be identified from this lognormal distribution, with $\operatorname{Pr}\left\{\xi_{j k} \leq \xi_{j k}^{l}\right\}=\operatorname{Pr}\left\{\xi_{j k} \geq \xi_{j k}^{u}\right\}=2.5 \%$. If $\xi_{j k}^{l} \leq 1 \leq \xi_{j k}^{u}$, there is insufficient evidence to reject the null hypothesis. Otherwise, $\mathrm{H}_{1}$ can be rejected, and it can be concluded that the relative effect of distraction type $j$ on the performance measure is $\left(\pi_{j k}-1\right) \times 100 \%$ greater than that of distraction type $k$ at the $5 \%$ significance level.

\subsection{Demographic Effects}

To analyze the demographic effect on the level of distraction type $k$, the set of driving performance factors $f_{k i}$ was divided into two groups according to the demographic factor, $\left(f_{k i}^{1}, \forall i \in G_{1}\right)$ and $\left(f_{k i}^{2}, \forall i \in G_{2}\right)$, where $G_{1}$ and $G_{2}$ are two demographic groups with $G_{1} \cap G_{2}=\phi$, i.e., an empty set, and $G_{1} \cup G_{2}=M$, i.e., a set comprising all of the participants. The demographic effects on certain factors were analyzed, including the participants' genders (female $=0$, male $=1$ ), ages ( 25 or above $=1$ versus below $25=0$ ), numbers of years with a full driving license (more than 3 years $=1$ versus 3 years or less $=0$ ), driving frequencies (more than once per week $=1$ versus once or less than once per week $=0$ ) and driving durations per week (more than 3 hours $=1$ versus 3 hours or less $=0$ ) and whether the participants had ever texted while driving (yes $=1$ versus no $=0$ ). Standard ANOVA analysis of these two sets of factors was then conducted to ascertain the demographic effect on the distraction level. 


\section{RESULTS}

The driving performance and cross-comparison distraction factors were analyzed under different distraction conditions.

\subsection{Driving Performance Factor}

The driving performance factor was used to evaluate the impairment effects of different distraction conditions, including reading Chinese (RC/ND), reading English (RE/ND), typing Chinese (TC/ND) and typing English (TE/ND), on the driving performance of young drivers compared with a no-distraction condition. The estimated mean $\mu_{k}$ and standard deviation $\sigma_{k}$ of the driving performance factor based on the different distraction types are summarized in Table 4.

\section{[Insert Table 4 here]}

Different distraction types were found to increase the performance measures, including RT, DLU and DSF, by $\left(\mu_{k}-1\right) \times 100 \%$ at the $5 \%$ significance level compared with the nodistraction condition. In contrast, no evidence was found for the driving distraction factor in relation to CFD.

The driving performance factor results indicated that the participants' RT increased under all of the distraction types: the reaction time of reading Chinese comparing to no distraction (RC/ND) increased 31\%, the reaction time of reading English comparing to no distraction (RE/ND) increased 51\%, the reaction time of typing Chinese comparing to no distraction (TC/ND) increased 95\%, and the reaction time of typing English comparing to no distraction (TE/ND) increased 76\%, all at 95\% significant level.

The driving performance factors of DLU and DSF similarly increased under the distraction conditions. The DSF performance results were more significant, as the performance factor doubled compared with the no-distraction condition: the driving speed fluctuation of reading Chinese comparing to no distraction (RC/ND) increased 92\%, the driving speed fluctuation of reading English comparing to no distraction (RE/ND) increased 108\%, the driving speed fluctuation of typing Chinese comparing to no distraction (TC/ND) increased 154\%, and average driving speed fluctuation of typing English comparing to no distraction (TE/ND) increased $144 \%$, all at 95\% significant level.

Among these driving performance measures, the DLU performance measure was the most sensitive, such that all of the performance factors increased by more than 3 times at least under the different distraction conditions compared with the no-distraction condition: the driving lane undulation of reading Chinese comparing to no distraction (RC/ND) increased 2.97 times, the driving lane undulation of reading English comparing to no distraction (RE/ND) increased 2.68 times, the driving lane undulation of typing Chinese comparing to no distraction (TC/ND) increased 8.72 times, and the driving lane undulation of typing English comparing to no distraction (TE/ND) increased 4.84 times, all at 95\% significant level.

As shown in Table 4, typing text messages and Chinese messages in particular distracted the participants more than reading the messages. The means of the driving performance factor of RT, DLU and DSF were all significantly greater than the other distraction conditions when 
the participants were typing Chinese. Typing English text messages was the second-largest distraction condition among all of the driving performance measures based on the means of the driving performance factors. However, more rigorous cross-comparison analyses were conducted to ascertain the relative effects of the different distraction factors, and their findings are detailed in the next section.

\subsection{Cross-Comparison Driving Distraction Factor}

The cross-comparison driving distraction factor was used to examine the degree of distraction severity under diverse conditions when (a) the same language is used in different distraction tasks, including typing Chinese versus reading Chinese (TC/RC) and typing English versus reading English (TE/RE), and (b) different languages are used in the same distraction task, including reading English versus reading Chinese (RE/RC) and typing English versus typing Chinese (TE/TC). The results of the cross-comparison driving distraction factors are presented in Table 5.

[Insert Table 5 here]

(a) Same language used for different distraction tasks

Table 5 compares typing and reading text messages in Chinese, and shows that the mean ratios for the reaction time (RT) of typing Chinese comparing to reading Chinese (TC/RC) increased 20\%, and driving speed fluctuation (DSF) of typing Chinese comparing to reading Chinese (TC/RC) increased 40\%, both are at 95\% significant level. Comparing typing and reading text messages in English reveals that the mean ratios for the reaction time (RT) of typing English comparing to reading English (TE/RE) increased 52\%, and driving speed fluctuation (DSF) of typing English comparing to reading English (TE/RE) increased 155\%, both are at 95\% significant level. Therefore, typing text messages while driving generally impaired driving performance more than reading messages, regardless of the language used.

(b) Different languages used for the same distraction task

English and Chinese were compared for the same distraction tasks. For the reading task, the mean ratio for the reaction time (RT) of reading English comparing to reading Chinese (RE/RC) increased 22\%, the mean ratio for driving lane undulation (DLU) of reading English comparing to reading Chinese (RE/RC) increased 106\%, and mean ratio for driving speed fluctuation (DSF) of reading English comparing to reading Chinese (RE/RC) increased 39\%, all at 95\% significant level. For the typing task, only the mean ratio for the car-following distance (CFD) of tying English comparing to typing Chinese (TE/TC) increased 50\%, at 95\% significant level. However, no evidence was found for the other driving performance measures between the different languages. The distraction effects between English and Chinese may be remarkably different for the reading distraction task and unremarkable for the typing task.

The CFD between cars was insignificant for the impairment measures of the driving performance and cross-comparison driving distraction factors.

\subsection{Demographic Effects}

The standard one-way ANOVA F-statistics of the demographic effects on the driving 
performance and cross-comparison distraction factors were determined to evaluate the effects of the participants' characteristics while driving under the distraction conditions. The analysis results are presented in Table 6. The prevalence of texting while driving was found to be associated with significant differences in reaction time for the no-distraction condition and the condition under which the participants read English text messages at the 1\% significance level. However, no evidence showed a significant difference in impairment under the other distraction conditions arising from the different driver characteristics.

[Insert Table 6 here]

\section{DISCUSSION}

\subsection{Impairment Effects of Text Messaging while Driving}

The results of this study demonstrated that the identified distraction performance measures, including RT, DLU and DSF, were significant impaired, and were therefore consistent with prior findings that text messaging while driving generally impairs driving performance (Hallett et al., 2012). In Hallett et al.'s online survey (2012), almost 80\% of the participants' response in the 'very unsafe' and 'moderately unsafe' categories for the behavior of reading or sending a text message while driving, and when comparing the responses to the "very unsafe' category, it appears that typing message is more unsafe than reading message in the participants' perception. In the data of participants only who admitted to text messaging while driving, 89.1\% (650 of 730) of participants responded "yes" to the question "if they felt that text messaging impairs their driving performance". The questionnaire survey result kept consistency with our simulated driving experiment study result that engaging in text messaging (reading and typing) is not safe, and impairs driving performance, in perception and maneuvering aspect.

Numerous studies have argued that dialing a phone or having a conversation have little to no effect on the deviations in a car's lateral position (Törnros and Bolling, 2005; Rudin-Brown et al., 2013). On the contrary, a study conducted by Hosking et al. (2009) demonstrated that a car's lateral position is highly impaired by text messaging behavior while driving, with the variability in lane position increasing by $50 \%$ and missed lane changes increasing by $140 \%$. Indeed, the results of this study complemented Hosking's finding that the driving lane undulation performance measure is the most sensitive measure, such that all of the performance factors under the different distraction types increase by more than three times compared with the no-distraction condition. This may be a result of steering wheel manipulation. In this study, the variability in lane position increased about 3 times when participants engaged in the unsafe driving activity of reading message while driving, both in Chinese and English; and typing Chinese and English increased 8.72 times and 4.84 times respectively according to no distraction condition, which is more severe than the previous study. Because using the hand-held mobile phones to read and type text messages may increase driving maneuvering difficulty, as this behavior required the participants to take one hand away from the steering wheel, and kept their attention to the mobile phone instead of the road driving condition, which might have made their driving performance severely unstable than just dialing or have conversation via mobile phone.

In terms of DSF, a study by Harrison et al. (2011) suggested that most students drift into other lanes and speed at $10 \mathrm{mph}$ over the speed limit while texting and driving, which reflects 
the impairment of their ability to maintain driving stability. A telephone survey conducted by Beck et al. (2007) revealed that a driver's speeding tendency increases under distraction conditions and especially while text messaging. In this study, the driving speed variation was found to increase when the participants texted while driving, which was consistent with previous studies. However, some research studies have found that texting while driving decreases vehicle speed because the distraction increases the driver's mental workload (Törnros and Bolling, 2005; Young et al., 2014; Rudin-Brown et al., 2013) and increases the difficulty of maneuver maintenance (Törnros and Bolling, 2005; Hosking et al., 2009; Drews et al., 2009; Beede and Kass, 2006) with compensation behavior, which may be affected by the limited experimental repetition times.

\subsection{Distraction Contributions of Text Messaging while Driving}

In this study, the distraction contributions of text messaging while driving were examined when (a) the same language was used to perform different distraction tasks (reading versus typing text messages) and (b) different languages were used to perform the same distraction task (English verses Chinese messages).

\subsubsection{Reading versus typing text messages}

In this study, both reading and typing text messages were found to degrade one's driving performance. Nevertheless, typing text messages seemed to distract the participants more than reading messages, and comparatively increased their RT and DSF.

Typing text messages while driving impairs driving maneuver maintenance more than reading messages. The mental workload and physical manipulations involved in the mobile phone typing procedure may cause this difference, as they impair drivers' cognition, decisionmaking ability and safe maneuvering in relation to their RT, DLU and DSF. The demands of the mental workload increase because the typing procedure is more complicated. The driver must process information when reading text messages, memorize the messages to figure out the reply content and organize the typing procedure using the specified vocabulary and sentences of the input system keyboard. However, the physical manipulations required to type text messages on a mobile phone while driving is even worse, as drivers have the complicated task of not only taking their eyes off the road and removing their hands from the steering wheel to focus on the mobile phone screen (Kawano et al., 2005; Owens et al. 2011), but also typing and inputting system procedures that make them divert their eyes from the road more frequently and for longer periods, resulting in an easier loss of steering control.

\subsubsection{English versus Chinese text messaging}

In this study, text messaging in either Chinese or English significantly impaired driving performance compared with a no-distraction condition. Due to the complexity of the Pinyin phonetic Chinese input system, which requires an additional step of character selection, Chinese text messaging was expected to have a more critical effect on impaired driving performance than English text messaging. Considering that all of the participants in the simulation study were native Chinese speakers and that Chinese is character based and requires fewer characters than English to produce the same content, the distraction effect from reading Chinese messages was expected to be weaker than that from reading English messages. The results surprisingly showed that the difference in impairment of the participants' driving performance under the distraction of typing text messages was not 
significant across the two languages. However, the difference in impairment caused by reading text messages between the two languages was remarkable.

The difference between typing text messages in Chinese and English was found to be unremarkable, which indicates an efficient and effective way for Hong Kong and other Chinese-writing jurisdictions to learn about the current experience of legislation and education enforcement strategies in English-writing jurisdictions. The legislation against the use of hand-held phones while driving has been in place in Hong Kong for a decade and has been found to effectively decrease hand-held phone conversations while driving (Road Safety Council, 2003). However, text messaging remains prevalent despite drivers' awareness of crash and injury risks due to the use of mobile phones while driving. More executive actions could be taken and improved through better administration, and sustainable safety education with targeted driver education campaigns should be introduced to decrease the frequency of text messaging while driving.

\section{CONCLUSION}

This study examined the distraction effects of text messaging using a driving simulator approach. In particular, it compared the distraction effects of reading or typing Chinese and English text messages. Driving performance factors including RT, DLU and DSF, which determine drivers' abilities to maintain driving performance under all of the distraction conditions, were significantly impaired compared with a no-distraction condition. However, the CFD between cars did not significantly change as a measurement factor compared with the no-distraction condition.

Twenty-six participants, half of whom had previously texted while driving, took part in the simulated driving experiment. The results are summarized as follows.

(1) Reading and writing text messages in both English and Chinese impaired driving performance.

(2) Typing text messages impaired driving performance more than reading messages regardless of language. Texting while driving led to speeding (average driving speed) on occasion compared with the no-distraction condition.

(3) Although the Chinese input system for text messages is more complicated, the text messages in both languages did not exhibit remarkable differences in terms of distraction.

The findings of this study have implications for the development of future road safety enforcement and education strategies. In terms of enforcement, stricter penalties are essential to deter the use of handheld mobile phones and text messaging. Furthermore, taking into account the complexity of the Pinyin phonetic Chinese input method, the distraction caused by typing Chinese messages could be stronger than that caused by typing English messages. This may be critical to the development of relevant enforcement strategies in Chinese communities. In terms of education, as mobile phone usage is popular among young drivers, who are likely to have limited driving experience and defensive driving skills, it is important to highlight how text messaging impairs driving performance and its association with traffic accident risk. Future studies could further explore the different levels of impairment due to distraction according to the language and relevant input method. 


\section{ACKNOWLEDGEMENTS}

This research was jointly supported by a research postgraduate studentship, a grant from the Research Grants Council of the Hong Kong Special Administrative Region, China (Project No. HKU 7188/11E) and a grant from the University Research Committee of the University of Hong Kong (No. 201309176024). We thank Liqiao Zhang for her help with the data collection.

\section{REFERENCES}

AL-DARRAB, I. A., KHAN, Z. A. \& ISHRAT, S. I. 2009. An experimental study on the effect of mobile phone conversation on drivers' reaction time in braking response. Journal of Safety Research, 40, 185-189.

ATCHLEY, P., ATWOOD, S. \& BOULTON, A. 2011. The choice to text and drive in younger drivers: Behavior may shape attitude. Accident Analysis \& Prevention, 43, 134-142.

AUBERLET, J.-M., ROSEY, F., ANCEAUX, F., AUBIN, S., BRIAND, P., PACAUX, M.-P. \& PLAINCHAULT, P. 2012. The impact of perceptual treatments on driver's behavior: From driving simulator studies to field tests-First results. Accident Analysis \& Prevention, 45, 9198.

BECK, K. H., YAN, F. \& WANG, M. Q. 2007. Cell phone users, reported crash risk, unsafe driving behaviors and dispositions: A survey of motorists in Maryland. Journal of Safety Research, 38, 683-688.

BEEDE, K. E. \& KASS, S. J. 2006. Engrossed in conversation: The impact of cell phones on simulated driving performance. Accident Analysis \& Prevention, 38, 415-421.

BELLA, F. 2008. Driving simulator for speed research on two-lane rural roads. Accident Analysis \& Prevention, 40, 1078-1087.

CHIANG, Y.-C., TUNG, C.-L. \& CHEN, T.-S. 2002. An intellectual Chinese input system for mobile phones based upon Chinese phonetic symbols. International Journal of Computer Processing of Languages, 15, 395-406.

CHRISTOFOROU, Z., KARLAFTIS, M. G. \& YANNIS, G. 2013. Reaction times of young alcoholimpaired drivers. Accident Analysis \& Prevention, 61, 54-62.

CONSIGLIO, W., DRISCOLL, P., WITTE, M. \& BERG, W. P. 2003. Effect of cellular telephone conversations and other potential interference on reaction time in a braking response. Accident Analysis \& Prevention, 35, 495-500.

DREWS, F. A., YAZDANI, H., GODFREY, C. N., COOPER, J. M. \& STRAYER, D. L. 2009. Text messaging during simulated driving. Human Factors: The Journal of the Human Factors and Ergonomics Society, 51, 762-770.

EDQUIST, J., RUDIN-BROWN, C. M. \& LENNÉ, M. G. 2012. The effects of on-street parking and road environment visual complexity on travel speed and reaction time. Accident Analysis \& Prevention, 45, 759-765.

FELDMAN, G., GREESON, J., RENNA, M. \& ROBBINS-MONTEITH, K. 2011. Mindfulness predicts less texting while driving among young adults: Examining attention- and emotionregulation motives as potential mediators. Personality and Individual Differences, 51, 856861.

GOODWIN, A. H., O’BRIEN, N. P. \& FOSS, R. D. 2012. Effect of North Carolina's restriction on teenage driver cell phone use two years after implementation. Accident Analysis \& Prevention, 48, 363-367.

HALLETT, C., LAMBERT, A. \& REGAN, M. A. 2012. Text messaging amongst New Zealand drivers: Prevalence and risk perception. Transportation Research Part F: Traffic Psychology and Behaviour, 15, 261-271.

HARRISON, M. A. 2011. College students' prevalence and perceptions of text messaging while driving. Accident Analysis \& Prevention, 43, 1516-1520. 
HARRISON, W. A. (1998). Applying Psychology to a Reluctant Road Safety: A Comment on South (1998). Australian Psychologist, 33(3), 238-240. doi: 10.1080/00050069808257413

HORREY, W. J. \& WICKENS, C. D. 2006. Examining the impact of cell phone conversations on driving using meta-analytic techniques. Human Factors, 48, 196-205.

HOSKING, S. G., YOUNG, K. L. \& REGAN, M. A. 2009. The effects of text messaging on young drivers. Human Factors: The Journal of the Human Factors and Ergonomics Society, 51, 582-592.

KAWANO, T., IWAKI, S., AZUMA, Y., MORIWAKI, T. \& HAMADA, T. 2005. Degraded voices through mobile phones and their neural effects: A possible risk of using mobile phones during driving. Transportation Research Part F: Traffic Psychology and Behaviour, 8, 331-340.

MAKISHITA, H. \& MATSUNAGA, K. 2008. Differences of drivers' reaction times according to age and mental workload. Accident Analysis \& Prevention, 40, 567-575.

MCCARTT, A. T., HELLINGA, L. A. \& BRATIMAN, K. A. 2006. Cell phones and driving: Review of research. Traffic Injury Prevention, 7, 89-106.

NAN, J., YUE, C., KAN, S., YE, L., YILONG, X., WATSON, B., FLEITER, J. KING, M., \& CHEN, C. (2011, 8-10 Aug. 2011). Big-five personality factors affecting driving behaviors. Paper presented at the Emergency Management and Management Sciences (ICEMMS), 2011 2nd IEEE International Conference on.

NELSON, E., ATCHLEY, P. \& LITTLE, T. D. 2009. The effects of perception of risk and importance of answering and initiating a cellular phone call while driving. Accident Analysis \& Prevention, 41, 438-444.

NEMME, H. E. \& WHITE, K. M. 2010. Texting while driving: Psychosocial influences on young people's texting intentions and behaviour. Accident Analysis \& Prevention, 42, 1257-1265.

NIKOLAEV, A. G., ROBBINS, M. J. \& JACOBSON, S. H. 2010. Evaluating the impact of legislation prohibiting hand-held cell phone use while driving. Transportation Research Part a-Policy and Practice, 44, 182-193.

OWENS, J. M., MCLAUGHLIN, S. B. \& SUDWEEKS, J. 2011. Driver performance while text messaging using handheld and in-vehicle systems. Accident Analysis \& Prevention, 43, 939947.

PATTEN, C. J. D., KIRCHER, A., ÖSTLUND, J. \& NILSSON, L. 2004. Using mobile telephones: cognitive workload and attention resource allocation. Accident Analysis \& Prevention, 36, 341-350.

RAY B. (2014, August 26). Distracted driving: Self-control may be more effective than banning texting. Retrieved from http://www.riskscience.umich.edu/distracted-driving-self-controlmay-effective-texting-ban/

REDELMEIER, D. A. \& TIBSHIRANI, R. J. 1997. Association between cellular-telephone calls and motor vehicle collisions. New England Journal of Medicine, 336, 453-458.

ROAD SAFETY COUNCIL 2003. Hong Kong Road Safety Review. Road Safety Research Committee, December 2003, Hong Kong.

RUDIN-BROWN, C. M., YOUNG, K. L., PATTEN, C., LENNÉ, M. G. \& CECI, R. 2013. Driver distraction in an unusual environment: Effects of text-messaging in tunnels. Accident Analysis \& Prevention, 50, 122-129.

STAVRINOS, D., JONES, J. L., GARNER, A. A., GRIFFIN, R., FRANKLIN, C. A., BALL, D., WELBURN, S. C., BALL, K. K., SISIOPIKU, V. P. \& FINE, P. R. 2013. Impact of distracted driving on safety and traffic flow. Accident Analysis \& Prevention, 61, 63-70.

TÖRNROS, J. E. B. \& BOLLING, A. K. 2005. Mobile phone use-Effects of handheld and handsfree phones on driving performance. Accident Analysis \& Prevention, 37, 902-909.

TRAFFIC AND TRANSPORT (2014). Women and Men in Hong Kong - Key Statistics (2014 Edition). Retrieved from http://www.statistics.gov.hk/pub/B11303032014AN14B0100.pdf

VAN DRIEL, C. J. G., HOEDEMAEKER, M. \& VAN AREM, B. 2007. Impacts of a congestion assistant on driving behaviour and acceptance using a driving simulator. Transportation Research Part F: Traffic Psychology and Behaviour, 10, 139-152.

VIOLANTI, J. M. \& MARSHALL, J. R. 1996. Cellular phones and traffic accidents: An epidemiological approach. Accident Analysis \& Prevention, 28, 265-270.

WALSH, S. P., WHITE, K. M., HYDE, M. K. \& WATSON, B. 2008. Dialling and driving: Factors 
influencing intentions to use a mobile phone while driving. Accident Analysis \& Prevention, 40, 1893-1900.

YAN, X., RADWAN, E. \& GUO, D. 2007. Effects of major-road vehicle speed and driver age and gender on left-turn gap acceptance. Accident Analysis \& Prevention, 39, 843-852.

YAN, X., RADWAN, E., GUO, D. \& RICHARDS, S. 2009. Impact of "Signal Ahead" pavement marking on driver behavior at signalized intersections. Transportation Research Part F: Traffic Psychology and Behaviour, 12, 50-67.

YANG, Q., OVERTON, R., HAN, L. D., YAN, X. \& RICHARDS, S. H. 2013. The influence of curbs on driver behaviors in four-lane rural highways-A driving simulator based study. Accident Analysis \& Prevention, 50, 1289-1297.

YOUNG, K. L., RUDIN-BROWN, C. M., PATTEN, C., CECI, R. \& LENNÉ, M. G. 2014. Effects of phone type on driving and eye glance behaviour while text-messaging. Safety Science, 68, 4754. 


\section{APPENDIX}

Consider a lognormal distribution with a mean $\mathrm{E}(x)=\bar{x}$ and a variance $\operatorname{Var}(x)=\sigma^{2}$. To define the confident interval of a lognormal distribution, the parameters of this distribution, $\mathrm{E}(\ln x)$ and $\operatorname{Var}(\ln x)$ can be estimated based on its mean and variance as follows. Expanding Taylor's series of $\ln x$ around the mean value $\bar{x}$, we have

$$
\ln x=\ln \bar{x}+\frac{1}{\bar{x}}(x-\bar{x})-\frac{1}{2 \bar{x}^{2}}(x-\bar{x})^{2}+\cdots .
$$

Taking expectation on both sides of (A1) and ignoring higher order terms, we can show that

$$
\mathrm{E}(\ln x) \cong \mathrm{E}(\ln \bar{x})+\frac{1}{\bar{x}} \mathrm{E}(x-\bar{x})-\frac{1}{2 \bar{x}^{2}} \mathrm{E}\left((x-\bar{x})^{2}\right)=\ln \bar{x}-\frac{\sigma^{2}}{2 \bar{x}^{2}},
$$

because $\ln \bar{x}$ is a constant, $\mathrm{E}(x-\bar{x})=0$, and $\mathrm{E}\left((x-\bar{x})^{2}\right)=\operatorname{Var}(x)$. Taking variance on both sides of (A1) and ignoring higher order terms, we can show that

$$
\operatorname{Var}(\ln x) \cong \operatorname{Var}(\ln \bar{x}-1)+\frac{1}{\bar{x}^{2}} \operatorname{Var}(x)=\frac{\sigma^{2}}{\bar{x}^{2}},
$$

because $\ln \bar{x}-1$ is a constant. Based on the parameters in (A2) and (A3), we can determine the $2.5 \%$ and $97.5 \%$ percentile values of the lognormal distribution, which forms the $95 \%$ confident interval. 
Table 1 Driving Performance Dependent Sample Variables, Description, and References

\begin{tabular}{|c|c|c|c|}
\hline $\begin{array}{l}\text { Variable } \\
\text { Classification }\end{array}$ & Variable & Description & Sample Reference \\
\hline Reaction Time & $\begin{array}{l}\text { Reaction time } \\
\quad(R T)\end{array}$ & $\begin{array}{l}\text { The time from a hazard to } \\
\text { the brake onset. }\end{array}$ & $\begin{array}{l}\text { Redelmeier \& Tibshirani } \\
\text { (1997); Makishita \& } \\
\text { Matsunaga (2008); } \\
\text { Edquist et al. (2012); } \\
\text { Consiglio et al. (2003); } \\
\text { Christoforou et al. (2013); } \\
\text { Hosking et al. (2009); } \\
\text { Beede \& Kass (2006) }\end{array}$ \\
\hline $\begin{array}{l}\text { Lateral } \\
\text { Control }\end{array}$ & $\begin{array}{l}\text { Driving lane } \\
\text { undulation } \\
\text { (DLU) }\end{array}$ & $\begin{array}{l}\text { Standard deviation of lateral } \\
\text { position. }\end{array}$ & $\begin{array}{l}\text { Horrey \& Wickens } \\
\text { (2006); Törnros \& } \\
\text { Bolling (2005); Owens et } \\
\text { al (2011); Hosking et al. } \\
\text { (2009); Auberlet et al. } \\
\text { (2012); Beede \& Kass } \\
\text { (2006); Stavrinos et al. } \\
\text { (2013) }\end{array}$ \\
\hline $\begin{array}{l}\text { Longitudinal } \\
\text { Control }\end{array}$ & $\begin{array}{l}\text { Driving speed } \\
\text { fluctuation } \\
\text { (DSF) } \\
\text { Car-following } \\
\text { distance (CFD) }\end{array}$ & $\begin{array}{l}\text { Distance to the rear bumper } \\
\text { of the lead vehicle. }\end{array}$ & $\begin{array}{l}\text { Al-Darrab et al. (2009); } \\
\text { Edquist et al. (2012); } \\
\text { Drews et al. (2009); } \\
\text { Törnros \& Bolling } \\
\text { (2005); Stavrinos et } \\
\text { al.(2013) }\end{array}$ \\
\hline
\end{tabular}


Table 2 Summary of the participant statistics (sample size $=26$ )

\begin{tabular}{|c|c|c|c|c|}
\hline & Range/attribute & Frequency & Mean & S.D. \\
\hline \multicolumn{5}{|l|}{ Gender } \\
\hline - Female & & 7 & & \\
\hline - Male & & 19 & & \\
\hline Age & Min: 22; Max: 33 & & 24.2 & 2.5 \\
\hline - $\quad$ Below 25 & & 19 & & \\
\hline - 25 or above & & 7 & & \\
\hline Years license held & Min: 0.5; Max: 11 & & 3.9 & 2.3 \\
\hline - $\quad 3$ years or less & & 10 & & \\
\hline - $\quad$ More than 3 years & & 16 & & \\
\hline \multicolumn{5}{|l|}{ Driving frequency } \\
\hline - $\quad$ Once or less per week & & 17 & & \\
\hline - More than once per week & & 9 & & \\
\hline Driving duration per week & Min: 0.25; Max: 14 & & 2.2 & 3.1 \\
\hline - 3 hours or less & & 20 & & \\
\hline - $\quad$ More than 3 hours & & 6 & & \\
\hline \multicolumn{5}{|l|}{ Has texted while driving } \\
\hline - $\quad$ No & & 18 & & \\
\hline - Yes & & 8 & & \\
\hline
\end{tabular}

S.D. - Standard deviation 
Table 3 Summary statistics of the collected data

\begin{tabular}{lcccc}
\hline & Mean & S.D. & Min & Max \\
\hline RT (sec) & 1.09 & 0.52 & 0.50 & 3.20 \\
DLU (m) & 0.20 & 0.22 & 0.00 & 1.10 \\
DSF (km/h) & 3.88 & 2.38 & 0.09 & 13.62 \\
CFD (m) & 37.49 & 8.49 & 9.09 & 55.65 \\
\hline RT: reaction time; DLU: driving lane undulation; DSF: driving speed fluctuation; CFD: car- \\
following distance between two vehicles.
\end{tabular}


Table 4 Results of the driving performance factor under different driving distraction types

\begin{tabular}{|c|c|c|c|c|c|}
\hline & & \multicolumn{4}{|c|}{ Driving performance factor $f_{k}$} \\
\hline & & \multirow[b]{2}{*}{ Mean $\mu_{k}$} & \multirow[b]{2}{*}{$\mathrm{SD} \sigma_{k}$} & \multicolumn{2}{|c|}{ 95\% confidence interval } \\
\hline & & & & Lower $f_{k}^{l}$ & Upper $f_{k}^{u}$ \\
\hline \multirow{4}{*}{$\mathrm{RC} / \mathrm{ND}$} & $\mathrm{RT}^{*}$ & 1.31 & 0.07 & 1.18 & 1.46 \\
\hline & DLU* & 3.97 & 0.90 & 2.48 & 6.04 \\
\hline & $\mathrm{DSF}^{*}$ & 1.92 & 0.43 & 1.21 & 2.90 \\
\hline & CFD & 1.02 & 0.06 & 0.90 & 1.14 \\
\hline \multirow{4}{*}{$\mathrm{RE} / \mathrm{ND}$} & $\mathrm{RT}^{*}$ & 1.51 & 0.10 & 1.33 & 1.71 \\
\hline & DLU* & 3.68 & 0.95 & 2.14 & 5.91 \\
\hline & DSF* $^{*}$ & 2.08 & 0.32 & 1.51 & 2.78 \\
\hline & CFD & 1.09 & 0.06 & 0.97 & 1.21 \\
\hline \multirow{4}{*}{ TC/ND } & $\mathrm{RT}^{*}$ & 1.95 & 0.19 & 1.60 & 2.35 \\
\hline & DLU* & 9.72 & 3.49 & 4.51 & 18.41 \\
\hline & DSF* $^{*}$ & 2.54 & 0.34 & 1.94 & 3.27 \\
\hline & CFD & 1.13 & 0.07 & 1.00 & 1.28 \\
\hline \multirow{4}{*}{ TE/ND } & $\mathrm{RT}^{*}$ & 1.76 & 0.14 & 1.50 & 2.04 \\
\hline & DLU* & 5.84 & 1.36 & 3.60 & 8.98 \\
\hline & $\mathrm{DSF}^{*}$ & 2.44 & 0.25 & 1.99 & 2.96 \\
\hline & CFD & 1.07 & 0.07 & 0.94 & 1.21 \\
\hline
\end{tabular}

*Significant at the 5\% level.

ND: no distraction; RC: reading Chinese; RE: reading English; TC: typing Chinese; TE: typing English; RT: reaction time; DLU: driving lane undulation; DSF: driving speed fluctuation; CFD: car-following distance between two vehicles. 
Table 5 Results of the cross-comparison distraction factor of driving performance

\begin{tabular}{|c|c|c|c|c|c|}
\hline & & \multicolumn{4}{|c|}{ Cross-comparison distraction factor $\xi_{j k}$} \\
\hline & & \multirow[b]{2}{*}{ Mean $\pi_{j k}$} & \multirow[b]{2}{*}{$\mathrm{SD} \omega_{j k}$} & \multicolumn{2}{|c|}{ 95\% confidence interval } \\
\hline & & & & Lower $f_{j k}^{l}$ & Upper $f_{j k}^{u}$ \\
\hline \multirow{4}{*}{ TC/RC } & $\mathrm{RT}^{*}$ & 1.20 & 0.08 & 1.05 & 1.37 \\
\hline & DLU & 1.20 & 0.20 & 0.85 & 1.63 \\
\hline & $\mathrm{DSF}^{*}$ & 1.40 & 0.15 & 1.13 & 1.72 \\
\hline & CFD & 2.05 & 0.89 & 0.80 & 4.36 \\
\hline \multirow{4}{*}{$\mathrm{TE} / \mathrm{RE}$} & $\mathrm{RT}^{*}$ & 1.52 & 0.15 & 1.24 & 1.85 \\
\hline & DLU* & 2.55 & 0.53 & 1.66 & 3.76 \\
\hline & $\mathrm{DSF}^{*}$ & 1.77 & 0.24 & 1.35 & 2.28 \\
\hline & CFD & 7.66 & 6.79 & 0.91 & 29.39 \\
\hline \multirow{4}{*}{$\mathrm{RE} / \mathrm{RC}$} & $\mathrm{RT}^{*}$ & 1.22 & 0.10 & 1.04 & 1.43 \\
\hline & DLU* & 2.06 & 0.30 & 1.54 & 2.71 \\
\hline & $\mathrm{DSF}^{*}$ & 1.39 & 0.13 & 1.16 & 1.66 \\
\hline & CFD & 1.07 & 0.21 & 0.72 & 1.54 \\
\hline \multirow{4}{*}{ TE/TC } & $\mathrm{RT}$ & 1.11 & 0.13 & 0.87 & 1.39 \\
\hline & DLU & 1.25 & 0.20 & 0.90 & 1.69 \\
\hline & DSF & 1.21 & 0.17 & 0.91 & 1.57 \\
\hline & CFD* & 1.50 & 0.26 & 1.05 & 2.08 \\
\hline
\end{tabular}

*Significant at the 5\% level.

ND: no distraction; RC: reading Chinese; RE: reading English; TC: typing Chinese; TE: typing English; RT: reaction time; DLU: driving lane undulation; DSF: driving speed fluctuation; CFD: car-following distance between two vehicles. 
Table 6 One-way ANOVA results of driving performance measures by driver characteristic

\begin{tabular}{|c|c|c|c|c|c|c|c|c|}
\hline \multicolumn{3}{|c|}{ F-statistics } & \multirow{2}{*}{$\begin{array}{c}\text { Gender } \\
0.041\end{array}$} & \multirow{2}{*}{$\begin{array}{l}\text { Age } \\
1.433\end{array}$} & \multirow{2}{*}{$\begin{array}{c}\text { Years } \\
\text { license } \\
\text { held }\end{array}$} & \multirow{2}{*}{$\begin{array}{c}\text { Driving } \\
\text { frequency }\end{array}$} & \multirow{2}{*}{$\begin{array}{c}\text { Driving } \\
\text { duration }\end{array}$} & \multirow{2}{*}{$\begin{array}{c}\text { Has texted } \\
\text { while } \\
\text { driving }\end{array}$} \\
\hline \multirow{16}{*}{$\begin{array}{c}\text { Driving } \\
\text { performance } \\
\text { factor }\end{array}$} & \multirow{4}{*}{ RC/ND } & RT & & & & & & \\
\hline & & DLU & 0.242 & 1.899 & 0.087 & 0.730 & 0.455 & 0.231 \\
\hline & & DSF & 0.129 & 0.120 & 3.001 & 0.059 & 0.550 & 1.023 \\
\hline & & CFD & 0.008 & 0.006 & 1.534 & 1.669 & 0.020 & 0.135 \\
\hline & \multirow{4}{*}{ RE/ND } & $\mathrm{RT}$ & 1.329 & 2.247 & 0.815 & $9.494^{* *}$ & 0.075 & 0.679 \\
\hline & & DLU & 0.096 & 0.005 & 0.902 & 0.001 & 0.386 & 0.784 \\
\hline & & DSF & 3.176 & 0.141 & 2.254 & 0.493 & 2.619 & 2.038 \\
\hline & & CFD & 0.759 & 0.425 & 0.471 & 0.108 & 0.110 & 0.058 \\
\hline & \multirow{4}{*}{ TC/ND } & $\mathrm{RT}$ & 0.312 & 0.632 & 0.252 & 0.786 & 0.001 & 0.152 \\
\hline & & DLU & 0.102 & 2.138 & 0.810 & 0.518 & 0.265 & 0.451 \\
\hline & & DSF & 1.087 & 0.000 & 2.336 & 0.039 & 2.311 & 1.011 \\
\hline & & CFD & 0.007 & 0.008 & 0.115 & 0.201 & 1.069 & 0.014 \\
\hline & \multirow{4}{*}{ TE/ND } & $\mathrm{RT}$ & 1.641 & 0.122 & 1.349 & 0.104 & 0.003 & 0.023 \\
\hline & & DLU & 0.071 & 0.213 & 0.141 & 0.684 & 0.355 & 0.012 \\
\hline & & DSF & 0.892 & 0.476 & 3.107 & 1.871 & 0.398 & 0.001 \\
\hline & & CFD & 0.492 & 0.281 & 0.059 & 0.897 & 1.404 & 0.005 \\
\hline
\end{tabular}

**1\% had text messaged while driving.

ND: no distraction; $R C$ : reading Chinese; RE: reading English; TC: typing Chinese; TE: typing English. 


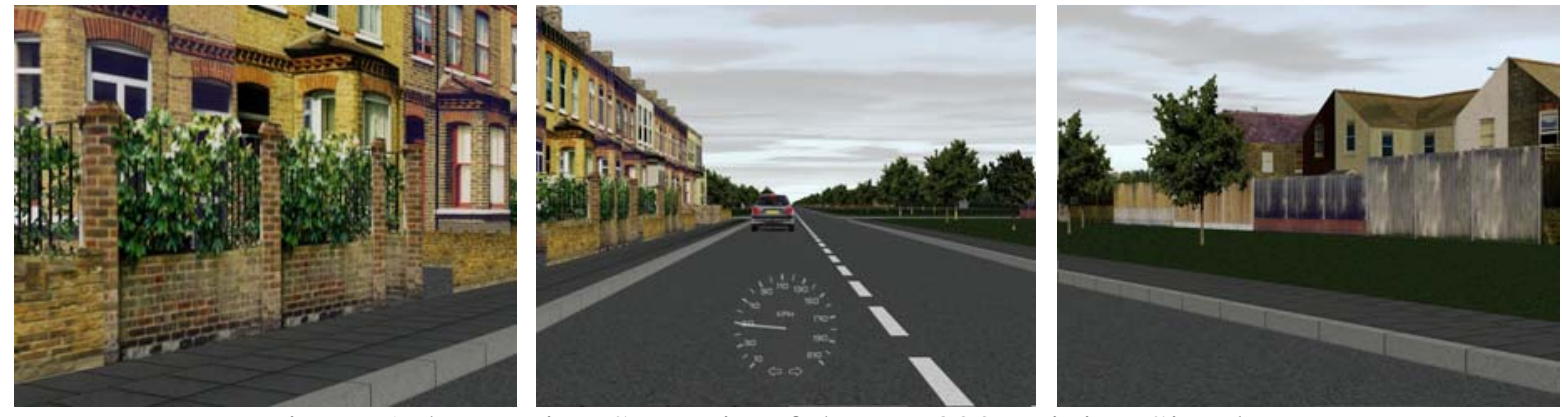

Figure 1 the Testing Scenario of the XP 300 Driving Simulator 


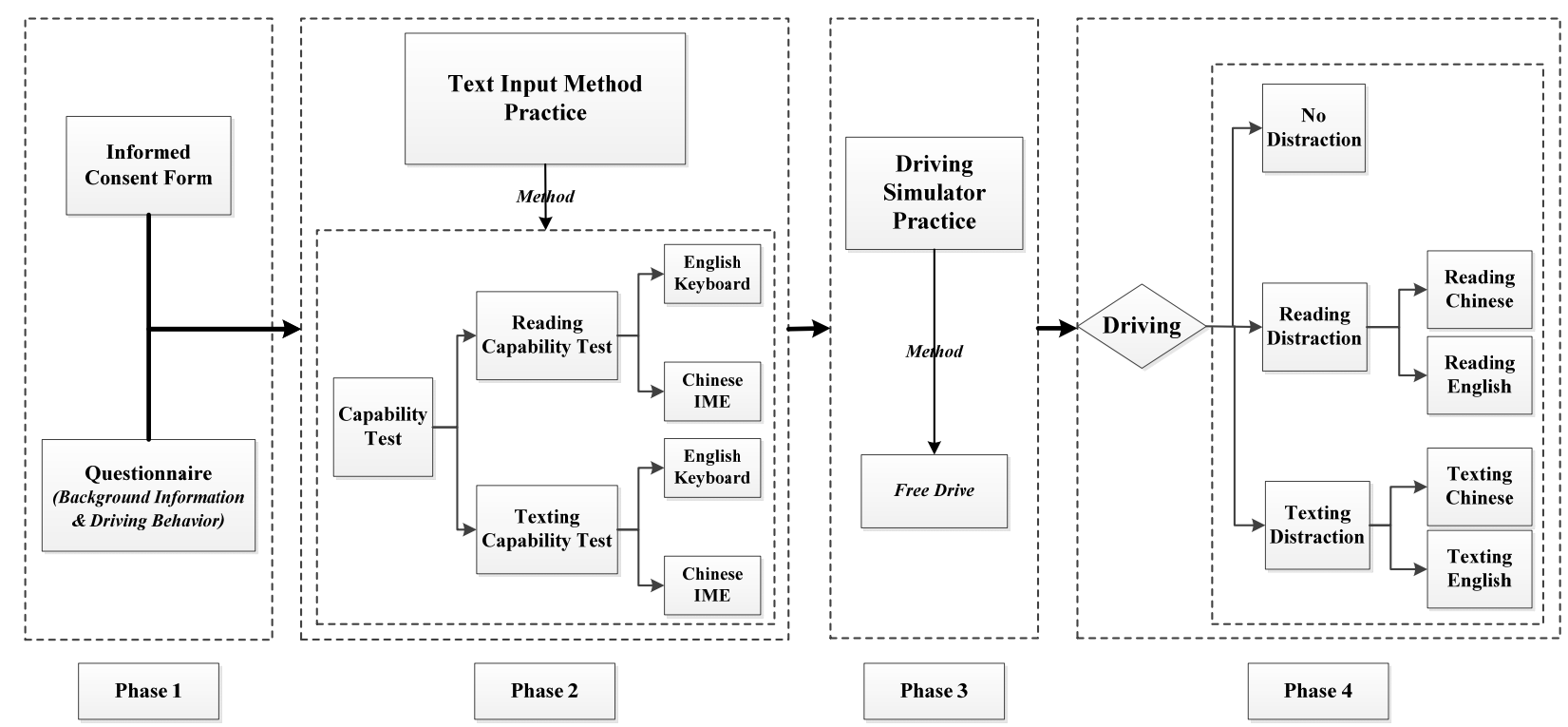

Figure 2 Driving simulator experiment procedure 